\title{
Autologous transplantation of adipose-derived mesenchymal stem cells attenuates cerebral ischemia and reperfusion injury through suppressing apoptosis and inducible nitric oxide synthase
}

\author{
DEHUA LI, YAN FANG, PAN WANG, WEI SHAN, ZHONGFU ZUO and LING XIE \\ Department of Anatomy, Liaoning Medical University, Jinzhou 121001, Liaoning Province, P.R. China
}

Received December 1, 2011; Accepted January 18, 2012

DOI: $10.3892 / \mathrm{ijmm} .2012 .909$

\begin{abstract}
The purpose of this study was to investigate whether autologous transplantation of adipose-derived mesenchymal stem cells (ADMSCs) has a neuroprotective effect against cerebral ischemia/reperfusion (I/R) injury in rats and to explore the possible underlying mechanisms. Adult male Sprague-Dawley rats were randomly assigned into the sham, I/R injury model (I/R), and model plus autologous transplantation of ADMSCs (ADMSC) groups. Cerebral $\mathrm{I} / \mathrm{R}$ injury was induced by $2 \mathrm{~h}$ middle cerebral artery occlusion (MCAO) followed by reperfusion for $24 \mathrm{~h}$. Rats in the $\mathrm{I} / \mathrm{R}$ and ADMSC groups were intravenously injected with culture medium and ADMSCs $\left(2.0 \times 10^{6}\right)$, respectively, at the onset of reperfusion and $12 \mathrm{~h}$ after reperfusion. Cerebral infarct volume was detected by triphenyltetrazolium chloride (TTC) staining. The histopathological changes and neuronal apoptosis in the ischemic penumbra were evaluated with $\mathrm{H} \& \mathrm{E}$ staining and the TUNEL assay, respectively. The nitric oxide (NO) content, caspase-3 activity and the Bax/Bcl-2 protein ratio were also measured. Moreover, the inducible nitric oxide synthase (iNOS) expression in the ischemic regions of rats was determined by immunohistochemical staining, quantitative real-time RT-PCR and western blot analysis. We found that autologous transplantation of ADMSCs significantly reduced the cerebral infarct volume, improved the I/R injury-induced brain damages and inhibited the neuronal apoptosis. ADMSC implantation also decreased caspase-3 activity and the Bax/ Bcl-2 protein ratio, and markedly downregulated the expression of iNOS and thus prevented NO release in response to cerebral I/R injury. Taken together, our results demonstrated that autologous transplantation of ADMSCs can protect the
\end{abstract}

Correspondence to: Professor Dehua Li, Department of Anatomy, Liaoning Medical University, no. 40, Section 3, Songpo Road, Jinzhou 121001, Liaoning Province, P.R. China

E-mail:1nmu_ldh@163.com

Key words: adipose-derived mesenchymal stem cells, cerebral ischemia and reperfusion injury, neuroprotection, apoptosis, inducible nitric oxide synthase brain against cerebral I/R injury via the inhibition of neuronal apoptosis and iNOS expression.

\section{Introduction}

Ischemic stroke is the third most frequent cause of death and the leading cause of serious and permanent disability worldwide in adults $(1,2)$. Although the underlying pathophysiology of ischemic stroke remains elusive, a defining feature of ischemic stroke is the ischemia/reperfusion (I/R) of cerebral blood vessels, leading to a decreased blood flow and irreversible neurocyte cellular damage $(3,4)$. In the last decade, considerable efforts have been made to develop a neuroprotective therapy for brain injury; however, intravenous recombinant tissue-plasminogen activator (t-PA), a thrombolytic agent, is the only licensed therapy for acute ischemic stroke, and only $1-2 \%$ of patients benefit from this intervention, largely as a consequence of the limited time window of administration and the risk of intracerebral hemorrhage $(5,6)$. A safe and effective therapeutic regimen for patients with ischemic stroke, therefore, is eagerly awaited.

Mesenchymal stem cells (MSCs) are a heterogeneous subset of stromal stem cells that reside in virtually all tissues and can differentiate into multilineage cells, such as adipocytes, chondrocytes, or osteoblasts $(7,8)$. MSC-based therapy has become a promising modern approach to treat various pathologies (9). For many years, bone marrow-derived MSCs (BMMSCs) were considered as an attractive candidate cell type for cell-based therapy and tissue engineering. However, harvesting BMMSCs is highly traumatic and yields very low number of cells, and these cells are difficult to expand in vitro. In contrast, adipose-derived MSCs (ADMSCs) have several distinct advantages of being abundant, easy to obtain with minimal invasiveness, and readily cultured to a sufficient number for autologous transplantation without the ethical issues of allografting (10). Recently, a growing body of experimental evidence has demonstrated the effectiveness of ADMSC-based therapy in improving end-organ ischemia or I/R injury (11-13). However, the possibility that autologous transplantation of ADMSCs may have a neuroprotective effect on cerebral I/R injury has not yet been examined.

In this study, we investigated the neuroprotective effect of ADMSCs in rats with 2-h middle cerebral artery occlusion 
(MCAO)/24 h reperfusion-induced brain injury. Furthermore, we tested the hypothesis that the neuroprotective effect of ADMSCs is associated with the inhibition of apoptosis and inducible nitric oxide synthase (iNOS) expression.

\section{Materials and methods}

Animals. Pathogen-free, adult male Sprague-Dawley rats weighing 200-250 g were obtained from the Experimental Animal Centre of Liaoning Medical University. They were housed in polycarbonate cages containing wood shaving and maintained in an air-conditioned room with free access to pelletted diet and water. The animal study protocol was reviewed and approved by the Animal Experimental Committee of Liaoning Medical University, and the animals received humane care in compliance with the Principles of Laboratory Animal Care.

Animal grouping and ADMSCs isolation. Forty-five rats were randomly assigned into the following three groups $(n=15$ for each group): the sham group, without MCAO and culture medium treated only; the I/R group, 2 h occlusion followed by $24 \mathrm{~h}$ reperfusion and culture medium treated; the ADMSC group, with MCAO and autologous ADMSCs implantation.

ADMSCs were isolated from adipose tissue of the rats in the ADMSC group as previously described with some modifications (14). Briefly, the rats were anesthetized with $10 \%$ chloral hydrate $(350 \mathrm{mg} / \mathrm{kg}$, i.p.) 14 days before the induction of I/R injury, and the subcutaneous adipose tissue was then obtained from the inguinal regions of these rats. The isolated adipose tissue was washed extensively with sterile phosphatebuffered saline (PBS) to remove contaminating debris and blood cells. The washed tissue was minced and digested with $0.1 \%$ collagenase (type I, Sigma-Aldrich, St. Louis, MO, USA) at $37^{\circ} \mathrm{C}$ in a water-bath shaker for $30 \mathrm{~min}$ with gentle agitation. Collagenase activity was neutralized with an equal volume of Dulbecco's modified Eagle's medium (DMEM; HyClone, Logan, UT, USA) containing 10\% fetal bovine serum (FBS; HyClone). Undigested tissue was removed by filtering twice through a $100-\mu \mathrm{m}$ and a $25-\mu \mathrm{m}$ mesh filter. The filtrate was centrifuged at $1,000 \times \mathrm{g}$ for $10 \mathrm{~min}$. The pellet was then resuspended in DMEM containing 10\% FBS and cultivated for $24 \mathrm{~h}$ at $37^{\circ} \mathrm{C}$ in $5 \% \mathrm{CO}_{2}$. After the unattached cells and debris were removed, fresh DMEM medium containing $10 \%$ FBS was added into the adherent cells, and the adherent cells were continuously cultured at $37^{\circ} \mathrm{C}$ in $5 \% \mathrm{CO}_{2}$ until $70-80 \%$ confluent.

ADMSCs characterization. Flow cytometry analysis was carried out to determine the identity of the isolated cells by assessing their surface markers. Briefly, the cultured ADMSCs cells (passage 2) were harvested and washed twice with cold PBS solution. After centrifugation and removal of the supernatants, the cells were resuspended and incubated with blocking buffer for $30 \mathrm{~min}$ at $4^{\circ} \mathrm{C}$. Following washing again in PBS, the cells were incubated for $30 \mathrm{~min}$ at $4^{\circ} \mathrm{C}$ in the dark with fluorescein isothiocyanate (FITC)-conjugated antibodies against CD29 (BD Biosciences, San Jose, CA, USA), CD34 (Novus Biologicals, Littleton, CO, USA), CD90 (Invitrogen, Carlsbad,
CA, USA), CD31 (Santa Cruz Biotechnology, Inc., Santa Cruz, CA, USA), CD45 (BD Biosciences) and CD13 (BioLegend, San Diego, CA, USA). Flow cytometry analysis was performed with a FACSCalibur cytometer (BD Biosciences) and data were analyzed using CellQuest software.

ADMSCs labeling for in vivo tracking. On Day 14, $30 \mathrm{~min}$ prior to ADMSCs implantation, the Vybrant CM-Dil celllabeling solution (Invitrogen, $50 \mu \mathrm{g} / \mathrm{ml}$ ) was added to the culture medium for ADMSCs labeling. The labeled cells were analyzed under a fluorescence microscope.

Animal model of cerebral I/R injury and autologous ADMSCs implantation. Cerebral I/R was induced by using the MCAO procedure as previously described (15). In brief, all rats were anesthetized with $10 \%$ chloral hydrate $(350 \mathrm{mg} / \mathrm{kg}$, i.p.). The right common carotid artery (CCA), the external carotid artery (ECA) and the internal carotid artery (ICA) were exposed via a midline neck incision. A 4-0 nylon monofilament with its tip rounded by heating over a flame and coated with silicone was introduced into the ECA lumen and carefully advanced into the ICA lumen until a slight resistance was felt, thereby to occlude the origin of the middle cerebral artery. Two hours after the induction of ischemia, the suture was slowly withdrawn to allow reperfusion. The rats in the sham group received all the surgical procedures but without the suture inserted. For the rats in the ADMSC group, intravenous infusion of autologous ADMSCs $\left(2.0 \times 10^{6}\right)$ was performed at the onset of reperfusion and $12 \mathrm{~h}$ after reperfusion via the tail vein. Animals in the sham and I/R groups received an equal volume of culture medium at each time point. All rats were anesthetized as described above and sacrificed by decapitation $24 \mathrm{~h}$ after reperfusion.

Measurement of cerebral infarct volume. The brains $(n=5)$ were rapidly removed after the decapitation, cut into six 2-mm thick coronal sections, and stained with $2 \%$ 2,3,5-triphenyltetrazolium chloride (TTC) solution at $37^{\circ} \mathrm{C}$ for $30 \mathrm{~min}$. Stained slides were washed in PBS solution for $5 \mathrm{~min}$ and immersed overnight in $4 \%$ paraformaldehyde. The brain unstained areas were defined as infarcted tissue, whereas normal tissue was stained red. The total infarct volume was presented as the sum of the infarct areas of the 6 sections and was presented as the percentage of cerebral ischemic volume in the ipsilateral hemispheric volume (16).

Histological evaluation. For histological evaluation, the brains were harvested and fixed in $4 \%$ paraformaldehyde at $4^{\circ} \mathrm{C}$. Samples were embedded in paraffin, sectioned at $5-\mu \mathrm{m}$ thickness with a microtome, and stained with hematoxylin and eosin (H\&E) for light microscopic examination.

Immunohistochemistry. The brains were fixed in $4 \%$ paraformaldehyde, embedded in paraffin, and cut into 5- $\mu \mathrm{m}$ sections. The sections were pretreated at $60^{\circ} \mathrm{C}$ for $1 \mathrm{~h}$, then dewaxed in xylene, hydrated, and washed in $0.01 \mathrm{~mol} / 1$ citrate buffer (pH 6.0). Following inhibition of endogenous peroxidase by $3 \% \mathrm{H}_{2} \mathrm{O}_{2}$ in methanol, the sections were incubated with a polyclonal antibody against rat inducible iNOS (1:200; Beijing Biosynthesis Biotechnology Co., Ltd., Beijing, China) over- 
night at $4^{\circ} \mathrm{C}$. After thoroughly washing with PBS solution, the corresponding secondary antibody was applied and incubated at room temperature for $30 \mathrm{~min}$. Reaction products were visualized by incubation with 3,3'-diaminobenzidine (DAB) and then counterstained with hematoxylin. Negative controls were achieved by substituting the primary antibody with isotypematched irrelevant antibody.

Terminal deoxynucleotidyl transferase dUTP nick-end labeling (TUNEL) staining. Internucleosomal DNA fragmentation was assessed on the paraffin-embedded sections by a standard TUNEL assay kit (Beyotime Institute of Biotechnology, Haimen, China). The sections $(n=5$, in each group) were prepared using the same method as the immunohistochemical analysis at $24 \mathrm{~h}$ after reperfusion. The staining was performed according to the manufacturer's recommended protocol. The cells displaying brown staining within the nucleus were counted as TUNEL-positive cells.

Measurements of caspase-3 activity and nitric oxide (NO). Levels of caspase-3 activity and NO in the ipsilateral hemisphere of ischemic brains were detected using a caspase-3 activity assay kit (Beyotime Institute of Biotechnology) and a colorimetric NO detection kit (Jiancheng Bioengineering Institute, Nanjing, China), respectively. These measurements were performed according to the respective manufacturer's instructions. The caspase-3 activity and NO content were normalized for total protein and then expressed as folds of the baseline of the sham group.

RNA isolation and quantitative real-time RT-PCR. Total-RNA was isolated from the ipsilateral hemisphere of ischemic brains using TRIzol reagent (Invitrogen) according to the manufacturer's instructions. Reverse transcription was performed with $1 \mu \mathrm{g}$ of total-RNA from each sample. Quantitative real-time RT-PCR was performed using SYBRGreen (Tiangen Biotechnology Co., Ltd., Beijing, China) on an Exicycler ${ }^{\mathrm{TM}} 96$ real-time quantitative thermal block (Bioneer, Daejeon, Korea). The PCR primer sequences were designed according to the rat iNOS and $\beta$-actin gene sequences reported in GenBank and were chemically synthesized: iNOS, forward, 5'-TCTCCCGAAACGCTACACTT-3' and reverse, 5'-CGTCTGGCGAAGAACAATC-3'; $\beta$-actin, forward, 5'-ACGTTGACATCCGTAAAGAC-3' and reverse, 5'-GAAGGTGGACAGTGAGGC-3'. The specificity of the PCR was confirmed by examining the dissociation reaction plot subsequent to real-time RT-PCR. $\beta$-actin served as the constitutive control. PCR reactions of each sample were conducted in triplicate. Data were analyzed through the comparative threshold cycle $\left(\mathrm{C}_{\mathrm{T}}\right)$ method (17).

Western blot analysis. Total proteins were extracted from the ipsilateral hemisphere of ischemic brains using radioimmunoprecipitation assay (RIPA) buffer (Beyotime Institute of Biotechnology), and protein concentrations were determined by using a bovine serum albumin (BSA) standard line. Equal amounts of protein were separated by sodium dodecyl sulfate polyacrylamide gel electrophoresis (SDS-PAGE) and then electrotransferred to polyvinylidene fluoride (PVDF) membranes. Membranes were blocked with 5\% skim milk at room tempera-
Table I. Cell surface phenotype of ADMSCs.

\begin{tabular}{lc}
\hline Cell surface markers & Positive rate $(\%)$ \\
\hline CD29 & $89.9 \pm 8.8$ \\
CD34 & $10.4 \pm 2.9$ \\
CD90 & $95.2 \pm 2.7$ \\
CD31 & $5.7 \pm 1.1$ \\
CD45 & $4.1 \pm 0.9$ \\
CD13 & $7.8 \pm 1.5$ \\
\hline
\end{tabular}

Values are the mean \pm SD. ADMSCs, adipose-derived mesenchymal stem cells.

ture for $2 \mathrm{~h}$ and then incubated overnight $\left(4^{\circ} \mathrm{C}\right)$ with anti-Bcl-2 (1:1,000; Abcam, Cambridge, MA, USA), anti-Bax (1:1,000, Santa Cruz Biotechnology, Inc.), or anti-iNOS (1:1,000; Beijing Biosynthesis Biotechnology Co., Ltd.) antibodies, followed by horseradish peroxidase-conjugated secondary antibodies. Protein bands were visualized with an ECL plus chemiluminescence kit (Millipore, Bedford, MA, USA).

Statistical analysis. All data are expressed as means \pm standard deviation (SD). Statistically significant differences between groups were determined by one-way ANOVA followed by the Bonferroni post hoc test. A P-value $<0.05$ was considered to be statistically significant.

\section{Results}

Flow cytometry analyses of cultured ADMSCs surface markers. The rat ADMSCs were isolated from the subcutaneous adipose tissue by standard procedures and grown for two passages in culture. Flow cytometry analyses demonstrated that ADMSCs expressed CD29 and CD90, but exhibited negligible expression of CD34, CD31, CD45 and CD13 (Table I). The surface marker expression profile of ADMSCs observed in this study is consistent with that in previous studies $(18,19)$.

Effects of ADMSCs transplantation on cerebral infarct volume and histological alterations. To explore whether ADMSCs transplantation has neuroprotective effects against cerebral I/R injury, we investigated the effects of ADMSCs transplantation on cerebral infarct volume by using TTC staining. In the I/R group, the infarct volume was $34.9 \pm 4.4 \%$ after $2 \mathrm{~h}$ MCAO followed by reperfusion for $24 \mathrm{~h}$. However, ADMSCs implantation resulted in a significant decrease in cerebral infarct volume after $2 \mathrm{~h} \mathrm{MCAO}$ and $24 \mathrm{~h}$ reperfusion $(\mathrm{P}<0.05)$ (Fig. 1A-D). In addition, under immunofluorescence microscopy, CM-Dil-labeled ADMSCs showing red fluorescence were found to be present in the infarct area (Fig. 1E), demonstrating that ADMSCs were capable of migrating to the infarct area within $24 \mathrm{~h}$ after venous injection.

The histopathological changes in ischemic regions of rats were evaluated at $24 \mathrm{~h}$ after reperfusion with H\&E staining. Rats in the sham group did not show any histopathological abnormalities and those in the I/R group exhibited marked cell damages, such as neuronal cell loss, pycnotic nucleus, and 

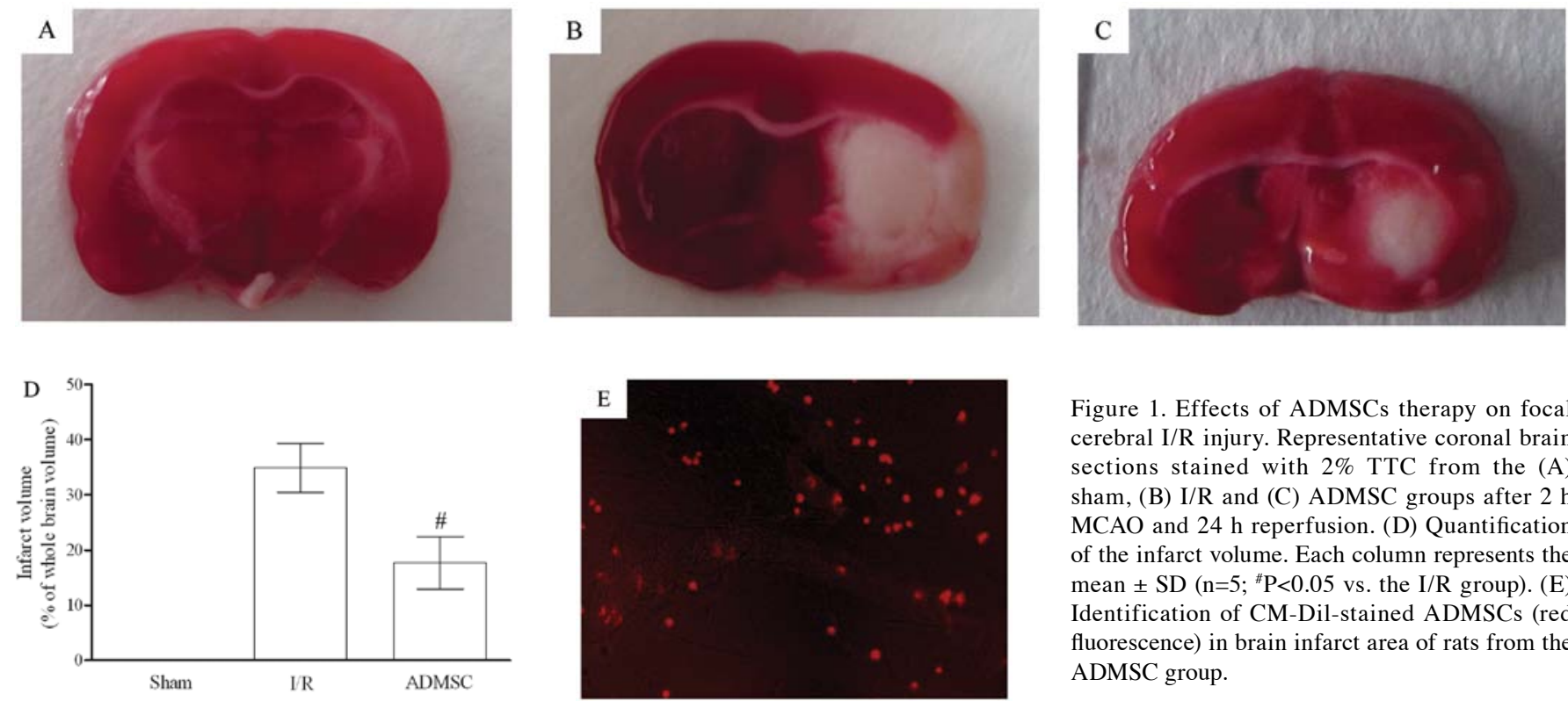

Figure 1. Effects of ADMSCs therapy on focal cerebral I/R injury. Representative coronal brain sections stained with $2 \%$ TTC from the (A) sham, (B) I/R and (C) ADMSC groups after $2 \mathrm{~h}$ $\mathrm{MCAO}$ and $24 \mathrm{~h}$ reperfusion. (D) Quantification of the infarct volume. Each column represents the mean $\pm \mathrm{SD}$ ( $\mathrm{n}=5 ;{ }^{\#} \mathrm{P}<0.05$ vs. the $\mathrm{I} / \mathrm{R}$ group). (E) Identification of CM-Dil-stained ADMSCs (red fluorescence) in brain infarct area of rats from the ADMSC group.
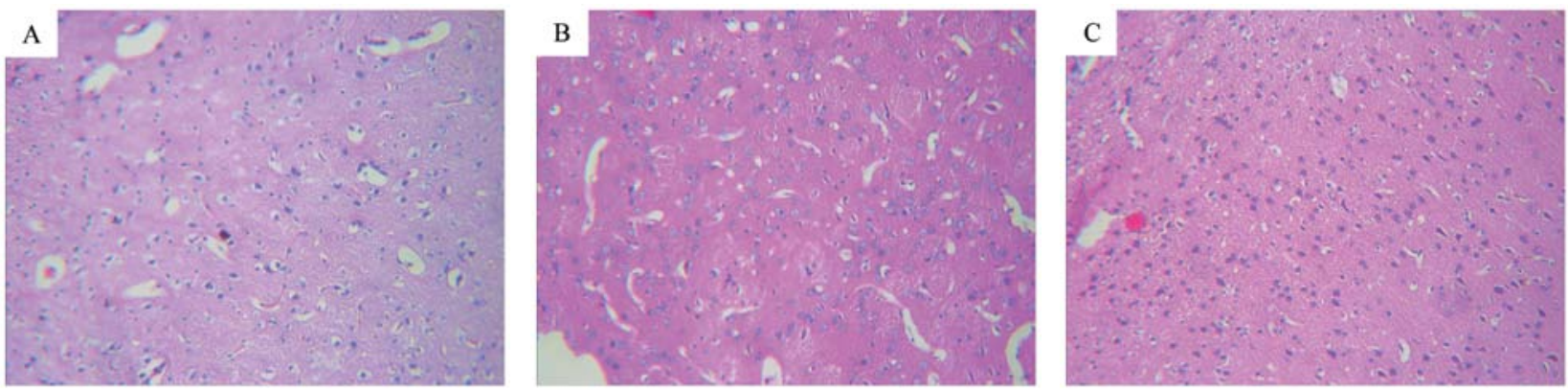

Figure 2. Effects of ADMSCs therapy on histopathological alterations in ischemic regions of the rat cortex induced by $2 \mathrm{~h}$ MCAO and $24 \mathrm{~h}$ reperfusion. Representative photomicrographs of brain sections stained by hematoxylin and eosin. (A) Sham group; (B) I/R group; (C) ADMSC group. (n=5; original magnification, x200).
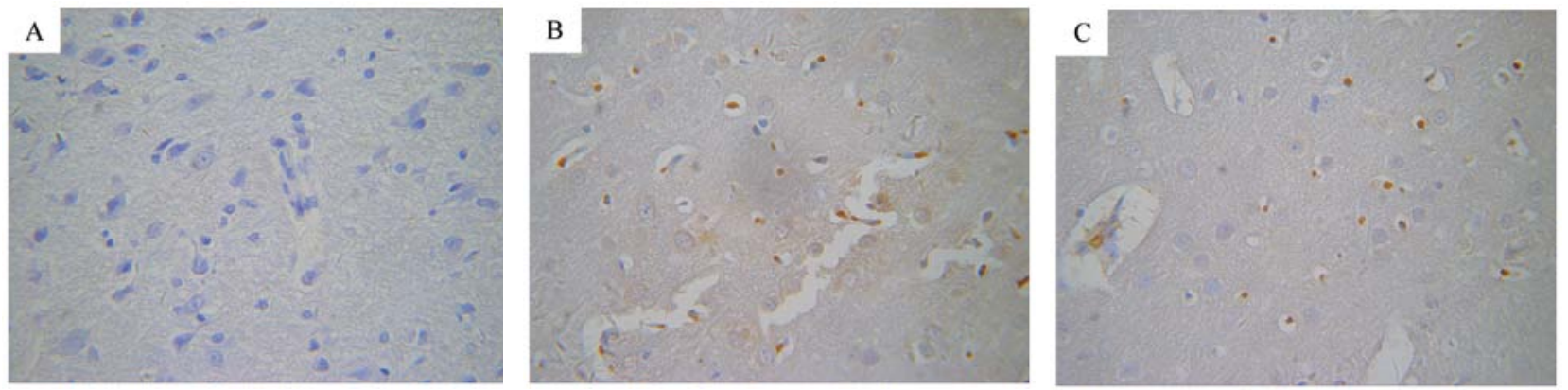

Figure 3. Effects of ADMSCs therapy on neuronal apoptosis in ischemic regions of the rat cortex after $2 \mathrm{~h}$ MCAO and $24 \mathrm{~h}$ reperfusion. Representative photomicrographs of brain sections stained for DNA breaks by the TUNEL assay. (A) Sham; (B) I/R; and (C) ADMSC groups. (n=5; original magnification, $x 400$ ).

dark staining of neurons (Fig. 2). In contrast, these damages were dramatically reduced by ADMSCs transplantation. Collectively, these findings suggest that autologous ADMSCs implantation is able to protect against brain damage induced by reperfusion after ischemia.

Effects of ADMSCs transplantation on TUNEL staining, caspase-3 activity, and the expression of Bcl-2 and Bax proteins. To determine whether ADMSCs transplantation exerts its neuroprotective effects against cerebral I/R injury by inhibiting neuronal apoptosis, we first assessed the typical DNA laddering pattern of neurons in the ischemic regions after reperfusion by TUNEL staining. For the rats in the sham group, the nuclei of neurons were almost negative for TUNEL staining (Fig. 3A). Abundant TUNEL-positive cells were found in the ischemic regions in rats with MCAO for 

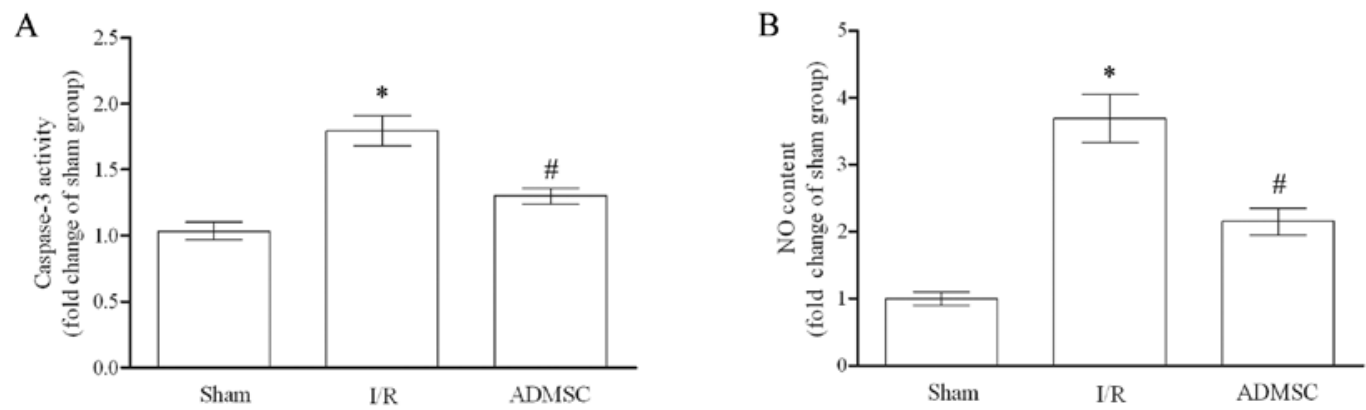

Figure 4. Effects of ADMSCs therapy on (A) caspase-3 activity and (B) NO content in ischemic regions of rat cortex after $2 \mathrm{~h}$ MCAO and $24 \mathrm{~h}$ reperfusion. Data are presented as the mean $\pm \mathrm{SD}\left(\mathrm{n}=5 ;{ }^{*} \mathrm{P}<0.05\right.$ vs. the sham group; ${ }^{*} \mathrm{P}<0.05$ vs. the $\mathrm{I} / \mathrm{R}$ group $)$.
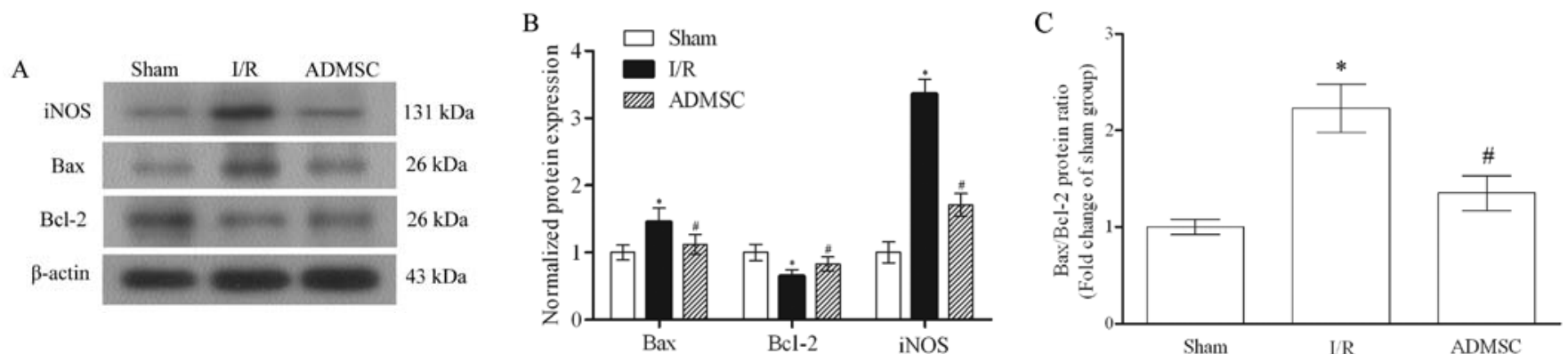

Figure 5. Western blot analysis of Bcl-2, Bax and iNOS protein levels in ischemic regions of the rat cortex induced by $2 \mathrm{~h}$ MCAO and $24 \mathrm{~h}$ reperfusion. (A) Representative blots are shown, and the protein size is expressed in $\mathrm{kDa}$. (B) Quantitative data are expressed as the intensity ratio of target proteins to $\beta$-actin. (C) Quantification of the ratio of Bax/Bcl-2 protein levels. ( $\mathrm{n}=5$; ${ }^{\mathrm{P}}<0.05$ vs. the sham group; ${ }^{*} \mathrm{P}<0.05$ vs. the I/R group).

$2 \mathrm{~h}$ followed by reperfusion for $24 \mathrm{~h}$ (Fig. 3B). However, the number of TUNEL-positive cells was markedly decreased in the ADMSC group (Fig. 3C).

Caspase- 3 is an essential protease for the execution of apoptosis, and activation of caspase- 3 is widely accepted as a reliable indicator for assessment of apoptosis (20). To provide more evidence for the ADMSCs-induced apoptosis reduction, we measured the enzymatic activity of caspase-3. As expected, the activity of caspase- 3 in the ischemic penumbra of rats with $2 \mathrm{~h}$ MCAO and $24 \mathrm{~h}$ reperfusion was markedly increased compared with the sham group, whereas the caspase- 3 activity was significantly decreased in the ADMSC group (Fig. 4A) $(\mathrm{P}<0.05)$.

The ratio of the pro-apoptotic Bax to the anti-apoptotic Bcl-2 is an important determinant of cellular susceptibility to apoptosis (21). We detected the ratio of $\mathrm{Bax} / \mathrm{Bcl}-2$ protein levels in the ischemic penumbra of rats with $2 \mathrm{~h}$ MCAO and $24 \mathrm{~h}$ reperfusion by western blot analysis. Cerebral I/R injury induced by MCAO resulted in upregulation of Bax and downregulation of $\mathrm{Bcl}-2$ protein levels, leading to an increase in the Bax/Bcl-2 ratio; however, these levels were significantly attenuated by autologous ADMSCs implantation $(\mathrm{P}<0.05)$ (Fig. 5). These data suggest that the inhibitory effect of ADMSCs implantation on brain I/R-induced apoptosis may be mediated, at least in part, by the regulation of $\mathrm{Bax}$ and $\mathrm{Bcl}-2$ expression.

Effects of ADMSCs transplantation on iNOS expression. NO produced by iNOS is a potent inflammatory mediator that contributes to the pathophysiology of cerebral I/R injury (22). Next, we focused on the effects of ADMSCs implantation on
NO levels in the ischemic penumbra of rats with $2 \mathrm{~h} \mathrm{MCAO}$ and $24 \mathrm{~h}$ reperfusion. NO levels were notably increased in the I/R model rats compared to the rats in the sham group (Fig. 4B). ADMSCs implantation significantly inhibited I/R-induced rise of NO levels in ischemic regions of rats, suggesting that ADMSCs implantation suppressed brain I/R-induced NO production.

Previous studies have demonstrated that the expression of iNOS is induced shortly after cerebral ischemia, thereby leading to excessive NO release (23). To investigate whether ADMSCs implantation suppresses NO production via the inhibition of iNOS expression, we further measured the expression levels of iNOS in ischemic penumbra regions after I/R injury. The photomicrographs of immunohistochemical localization of iNOS in ischemic penumbra are illustrated in Fig. 6A-C. In the sham group, positive signals of iNOS were observed weakly in a small number of macrophages. After MCAO for $2 \mathrm{~h}$ and reperfusion for $24 \mathrm{~h}$, positive signals of iNOS were observed prominently in neurons, endothelial cells, and microglia. However, ADMSCs implantation significantly attenuated the rise of brain I/R-induced iNOS expression.

To further determine the expression of iNOS in ischemic penumbra regions, iNOS mRNA and protein levels were measured by quantitative real-time RT-PCR and western blot analysis, respectively. Compared with the sham group, there was a marked increase in iNOS mRNA levels in the I/R group. ADMSCs implantation markedly attenuated the increase of iNOS mRNA in the ischemic penumbra of rats with $2 \mathrm{~h} \mathrm{MCAO}$ and $24 \mathrm{~h}$ reperfusion (Fig. 6D). Meanwhile, changes observed by western blot analysis were in accordance with the observations in the quantitative real-time RT-PCR 

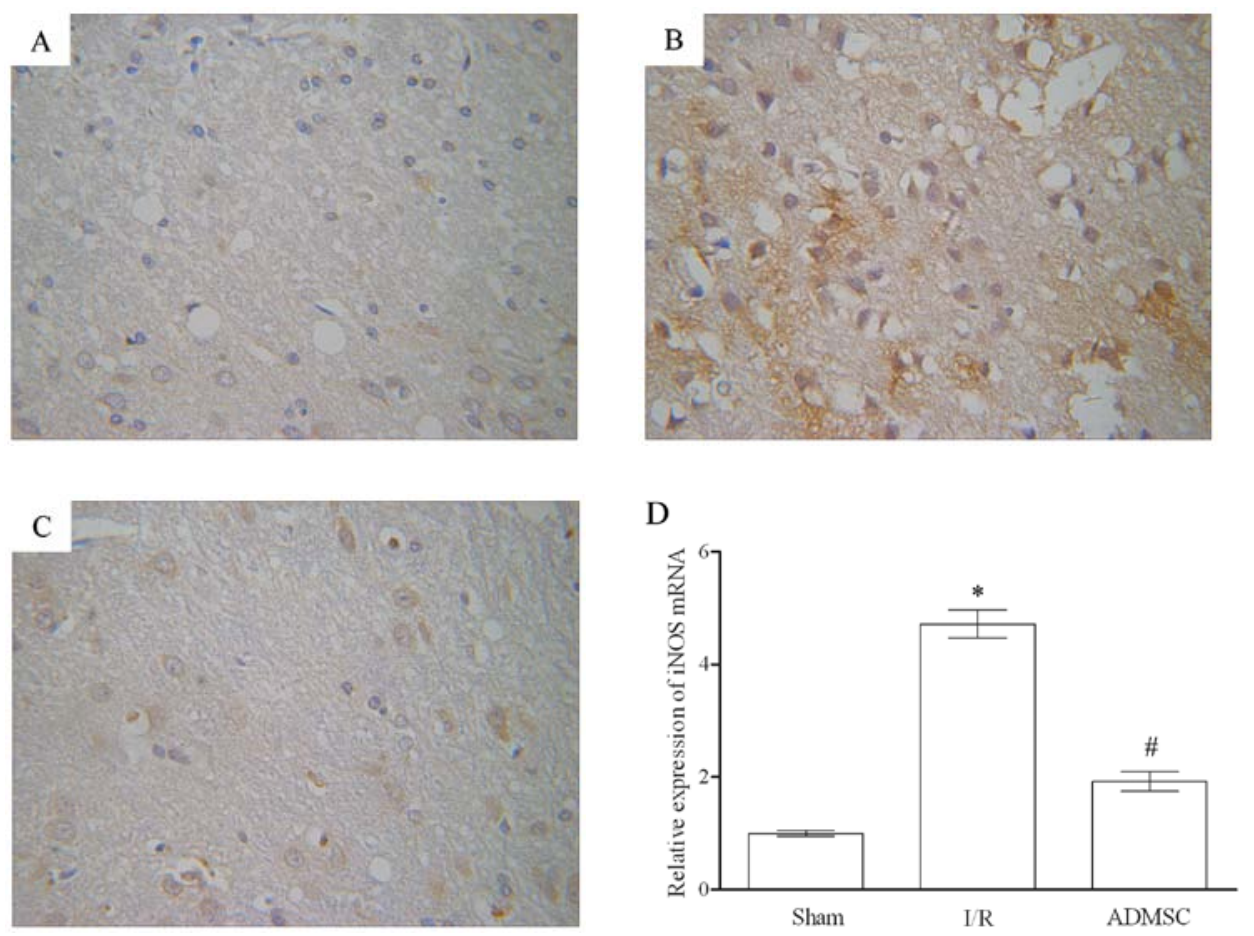

Figure 6. Effects of ADMSCs therapy on the expression of iNOS in ischemic regions of rat cortex induced by $2 \mathrm{~h}$ MCAO and $24 \mathrm{~h}$ reperfusion as shown by (A-C) immunohistochemical staining and (D) quantitative real-time RT-PCR. (A) Sham group; (B) I/R group; (C) ADMSC group. Original magnification, $\mathrm{x} 400$. (D) Data represent the mean $\pm \mathrm{SD}$. ( $\mathrm{n}=5 ;{ }^{*} \mathrm{P}<0.05$ vs. the sham group; ${ }^{*} \mathrm{P}<0.05$ vs. the $\mathrm{I} / \mathrm{R}$ group).

study (Fig. 5A and B). These findings indicate that ADMSCs implantation suppresses NO production via the inhibition of iNOS expression in cerebral I/R injury.

\section{Discussion}

The present study investigated, for the first time, the therapeutic effects of autologous ADMSCs implantation on cerebral I/R injury in a rat MCAO model. We found that ADMSCs implantation has potent neuroprotective effects against cerebral I/R injury, as evidenced by the remarkable decrease in cerebral infarct volume and the attenuation of neuronal loss and nuclei shrinkage. Meanwhile, ADMSCs implantation effectively inhibited neuronal apoptosis and reduced iNOS expression in the penumbra cortex of I/R rats, suggesting the possible mechanisms whereby ADMSCs implantation exerts its neuroprotective effects against cerebral I/R injury.

Infarction volume is an important imaging index for assessing the consequences of ischemic brain injury which leads to extensive neuronal damage (24). In the present study, cerebral I/R injury induced a prominent infarct size along with significant brain damages. ADMSCs implantation not only reduced the infarct size but also attenuated the neuronal damages. Our results are in agreement with the findings of Leu et al (11) who found that intravenous administration of ADMSCs into ischemic stroke rats significantly reduced brain infarct volume and remarkably improved the recovery of neurological function. In addition, several recent investigations also reported that autologous transplantation of ADMSCs can effectively attenuate acute pulmonary I/R injury and kidney I/R injury through its antioxidant, antiinflammatory and anti-apoptotic effects $(12,13)$. Our findings not only add to a growing body of evidence demonstrating the effectiveness of ADMSCs administration in the treatment of ischemic-related organ dysfunction, but are also worthy of future in-depth studies to evaluate the safety of ADMSCs therapy.

Previous studies have revealed that apoptosis is a major mechanism involved in the cerebral I/R injury-induced neuronal damages (25). In vitro studies have demonstrated that hypoxia in the brain following focal and global ischemia can trigger programmed cell death $(26,27)$. Herein, a significant number of TUNEL-positive cells were observed in the ischemic regions in rats with $\mathrm{MCAO}$ for $2 \mathrm{~h}$ followed by reperfusion for 24 h. Caspase-3 is well-known as an executioner of apoptosis, and there is a significant body of evidence that activation of caspase-3 is observed after ischemic injury. In the present study, we consistently found that the activity of caspase- 3 was markedly increased in the ischemic penumbra of rats with $2 \mathrm{~h}$ MCAO and $24 \mathrm{~h}$ reperfusion. The ratio of pro-apoptotic to anti-apoptotic Bcl-2 family members is thought to be a critical factor in regulating susceptibility to programmed cell death (21). In this study, we found that the ischemic penumbra in the I/R group exhibited an increased ratio of $\mathrm{Bax} / \mathrm{Bcl}-2$ at the protein level. Nonetheless, all these changes were significantly reversed by intravenous administration of ADMSCs. Our data are consistent with several recent reports showing that restoration of the levels of some apoptotic markers, such as caspase-3, Bax and Bcl-2, play an essential role in the protective effects of ADMSCs therapy against I/R injury $(12,13)$. Altogether, our findings not only corroborated previous reports that apoptosis produces neuronal death after I/R injury, but also provided evidence that ADMSCs implantation is able to prevent the apoptosis of neurons. 
NO is an unstable nitrogen radical that plays a crucial role in the pathophysiology of cerebral ischemic injury $(28,29)$. It is synthesized by three isoforms of NO synthase (NOS): neuronal (nNOS), endothelial (eNOS), and iNOS isoforms (30). Although the precise role of $\mathrm{NO}$ in ischemia remains controversial, it is generally accepted that NO has a dual role in the pathophysiology of cerebral ischemia. NO produced by nNOS and iNOS is neurotoxic by the formation of peroxinitrite free radical, leading to direct damage to mitochondrial enzyme and DNA (31-33). In contrast, NO derived from eNOS is beneficial in cerebral ischemia injury through its anti-inflammatory and anti-thrombotic effects $(34,35)$. Earlier studies have documented that the expression of iNOS is induced shortly after cerebral ischemia, thereby leading to excessive NO release (23). In this study, we found that ADMSCs implantation greatly reduced cerebral I/R injuryinduced increment of iNOS expression and subsequent NO production. This result provided evidence that the neuroprotective effects of ADMSCs implantation cerebral I/R injury may be mediated, at least in part, by inhibition of the inflammatory immune reaction injury through the downregulation of iNOS. Nonetheless, the exact mechanism by which ADMSCs therapy inhibits the expression of iNOS needs to be further elucidated.

In conclusion, we found that ADMSCs therapy could reduce cerebral infarct volume and improve neuronal damages in a rat model of MCAO-induced ischemia reperfusion brain injury. Further experiments demonstrated that this neuroprotective effect may be partly due to the inhibition of iNOS expression and neuronal apoptosis. Thus, autologous transplantation of ADMSCs may provide a novel therapeutic approach for stroke.

\section{Acknowledgements}

This study was supported by a grant from the Natural Science Foundation of Liaoning Province (grant no. 20102138).

\section{References}

1. Donnan GA, Fisher M, Macleod M and Davis SM: Stroke. Lancet 371: 1612-1623, 2008.

2. Roger VL, Go AS, Lloyd-Jones DM, et al: Heart disease and stroke statistics - 2011 update: a report from the American Heart Association. Circulation 123: e18-e209, 2011.

3. Woitzik J, Back T and Thome C: Flow-dependent versus spreading-like impairment of brain tissue integrity during focal cerebral ischemia and its consequences for neuroprotective strategies. Front Biosci 13: 1500-1506, 2008.

4. Li Q, Zhang R, Guo YL and Mei YW: Effect of neuregulin on apoptosis and expressions of STAT3 and GFAP in rats following cerebral ischemic reperfusion. J Mol Neurosci 37: 67-73, 2009.

5. Ginsberg MD: Neuroprotection for ischemic stroke: past, present and future. Neuropharmacology 55: 363-389, 2008.

6. Goldstein LB and Rothwell PM: Advances in prevention and health services delivery 2009. Stroke 41: e71-e73, 2010.

7. Pittenger MF, Mackay AM, Beck SC, et al: Multilineage potential of adult human mesenchymal stem cells. Science 284: 143-147, 1999.

8. Liu ZJ,Zhuge Y and Velazquez OC: Trafficking and differentiation of mesenchymal stem cells. J Cell Biochem 106: 984-991, 2009.

9. Ma T: Mesenchymal stem cells: from bench to bedside. World J Stem Cells 2: 13-17, 2010.

10. Locke M, Windsor J and Dunbar PR: Human adipose-derived stem cells: isolation, characterization and applications in surgery. ANZ J Surg 79: 235-244, 2009.
11. Leu S, Lin YC, Yuen CM, et al: Adipose-derived mesenchymal stem cells markedly attenuate brain infarct size and improve neurological function in rats. J Transl Med 8: 63, 2010.

12. Chen YT, Sun CK, Lin YC, et al: Adipose-derived mesenchymal stem cell protects kidneys against ischemia-reperfusion injury through suppressing oxidative stress and inflammatory reaction. J Transl Med 9: 51, 2011.

13. Sun CK, Yen CH, Lin YC, et al: Autologous transplantation of adipose-derived mesenchymal stem cells markedly reduced acute ischemia-reperfusion lung injury in a rodent model. J Transl Med 9: 118, 2011.

14. Zuk PA, Zhu M, Ashjian P, et al: Human adipose tissue is a source of multipotent stem cells. Mol Biol Cell 13: 4279-4295, 2002.

15. Longa EZ, Weinstein PR, Carlson S and Cummins R: Reversible middle cerebral artery occlusion without craniectomy in rats. Stroke 20: 84-91, 1989.

16. Swanson RA, Morton MT, Tsao-Wu G, Savalos RA, Davidson C and Sharp FR: A semiautomated method for measuring brain infarct volume. J Cereb Blood Flow Metab 10: 290-293, 1990.

17. Livak KJ and Schmittgen TD: Analysis of relative gene expression data using real-time quantitative PCR and the 2(-Delta Delta C(T)) Method. Methods 25: 402-408, 2001.

18. Strem BM, Hicok KC, Zhu M, et al: Multipotential differentiation of adipose tissue-derived stem cells. Keio J Med 54: 132-141, 2005.

19. Wang L, Deng J, Tian W, et al: Adipose-derived stem cells are an effective cell candidate for treatment of heart failure: an MR imaging study of rat hearts. Am J Physiol Heart Circ Physiol 297: H1020-H1031, 2009.

20. Lamkanfi M, Festjens N, Declercq W, Vanden Berghe T and Vandenabeele P: Caspases in cell survival, proliferation and differentiation. Cell Death Differ 14: 44-55, 2007.

21. Burlacu A: Regulation of apoptosis by Bcl-2 family proteins. J Cell Mol Med 7: 249-257, 2003.

22. ArunaDevi R, Ramteke VD, Kumar S, et al: Neuroprotective effect of s-methylisothiourea in transient focal cerebral ischemia in rat. Nitric Oxide 22: 1-10, 2010.

23. Samdani AF, Dawson TM and Dawson VL: Nitric oxide synthase in models of focal ischemia. Stroke 28: 1283-1288, 1997.

24. Lin TN, He YY, Wu G, Khan M and Hsu CY: Effect of brain edema on infarct volume in a focal cerebral ischemia model in rats. Stroke 24: 117-121, 1993.

25. Xing $\mathrm{B}$, Chen $\mathrm{H}$, Zhang $\mathrm{M}$, et al: Ischemic postconditioning inhibits apoptosis after focal cerebral ischemia/reperfusion injury in the rat. Stroke 39: 2362-2369, 2008.

26. Krajewski S, Mai JK, Krajewska M, Sikorska M, Mossakowski MJ and Reed JC: Upregulation of bax protein levels in neurons following cerebral ischemia. J Neurosci 15: 6364-6376, 1995.

27. Chen J,Zhu RL, Nakayama M, et al: Expression of the apoptosiseffector gene, Bax, is upregulated in vulnerable hippocampal CA1 neurons following global ischemia. J Neurochem 67: 64-71, 1996.

28. Iadecola C, Pelligrino DA, Moskowitz MA and Lassen NA: Nitric oxide synthase inhibition and cerebrovascular regulation. J Cereb Blood Flow Metab 14: 175-192, 1994.

29. Li H and Forstermann U: Nitric oxide in the pathogenesis of vascular disease. J Pathol 190: 244-254, 2000.

30. Marletta MA: Nitric oxide synthase: aspects concerning structure and catalysis. Cell 78: 927-930, 1994.

31. Huang Z, Huang PL, Panahian N, Dalkara T, Fishman MC and Moskowitz MA: Effects of cerebral ischemia in mice deficient in neuronal nitric oxide synthase. Science 265: 1883-1885, 1994.

32. Zhao X, Haensel C, Araki E, Ross ME and Iadecola C: Genedosing effect and persistence of reduction in ischemic brain injury in mice lacking inducible nitric oxide synthase. Brain Res 872: 215-218, 2000.

33. Sims NR and Anderson MF: Mitochondrial contributions to tissue damage in stroke. Neurochem Int 40: 511-526, 2002.

34. Zhang F and Iadecola C: Reduction of focal cerebral ischemic damage by delayed treatment with nitric oxide donors. J Cereb Blood Flow Metab 14: 574-580, 1994.

35. Huang Z, Huang PL, Ma J, et al: Enlarged infarcts in endothelial nitric oxide synthase knockout mice are attenuated by nitro-Larginine. J Cereb Blood Flow Metab 16: 981-987, 1996. 